\title{
Quantifying intrinsic chemical reactivity of molecular structural features for protein binding and reactive toxicity, using the MOSES chemoinformatics system
}

\author{
Johannes AH Schwöbel ${ }^{1 *}$, Bruno Bienfait ${ }^{1}$, Johann Gasteiger ${ }^{1}$, Thomas Kleinöder ${ }^{1}$, Jörg Marusczyk', Oliver Sacher ${ }^{1}$, \\ Christof H Schwab ${ }^{1}$, Aleksey Tarkhov ${ }^{1}$, Lothar Terfloth ${ }^{1}$, Mark TD Cronin ${ }^{2}$
}

From 7th German Conference on Chemoinformatics: 25 CIC-Workshop

Goslar, Germany. 6-8 November 2011

Covalent binding of xenobiotic compounds to endogenous biomolecular sites, e.g. protein residues, leads to potentially irreversible toxic effects such as enhanced acute toxicity or skin sensitization [1]. This mechanistic knowledge provides the basis for the in silico prediction of these toxicities, as required by the EU REACH legislation and the EU Cosmetics Directive. A general toxicity prediction can be based on three consecutive steps [2]: (1.) Identification of a potential reactive protein binding mechanism via a set of molecular structural features. Those structural features can be encoded by the Chemical Subgraph Representation Markup Language (CSRML), that supports a flexible annotation of meta information, including physicochemical properties as annotations. (2.) Confirmation and quantification of (bio)chemical reactivity. The potential for a chemical to be reactive can be captured by mechanistically based QSAR models. This intrinsic reactivity is calculated rapidly by descriptors of the chemoinformatics platform Molecular Structure Encoding System (MOSES) [3]. It represents electronic, steric and resonance effects in chemical structures. The performances obtained by these reactivity models are close to time-consuming quantum chemical reactivity calculations, e.g., $s e=0.44$ versus 0.40 for glutathione adduct formation via Michael addition, comparing predicted values to an experimental reactivity dataset [1]. (3.) Establishing a relationship between calculated reactivity and toxicity. The predicted

* Correspondence: schwoebel@molecular-networks.com

${ }^{1}$ Molecular Networks GmbH, Henkestraße 91, D-91052 Erlangen, Germany Full list of author information is available at the end of the article intrinsic reactivity values were linked to the computational prediction for different modes of toxic action, with good correlations between predicted and in vitro toxicity (up to $r^{2}=0.86$ ).

The combined use of structural information and computed reactivity could assist in the non-animal based risk assessment of chemicals for regulatory purposes and in the application of integrated testing strategies (ITS). The research has received funding from the EU FP7 COSMOS Project (grant agreement $n^{\circ} 266835$ ) and financing from COLIPA.

\section{Author details}

'Molecular Networks GmbH, Henkestraße 91, D-91052 Erlangen, Germany ${ }^{2}$ School of Pharmacy and Chemistry, Liverpool John Moores University, Byrom Street, Liverpool, L3 3AF, UK.

\section{Published: 1 May 2012}

References

1. Schwöbel JAH, et al: Measurement and estimation of electrophilic reactivity for predictive toxicology. Chem Rev 2011, 111:2562-2596.

2. Schwöbel JAH, et al: Application of a computational model for Michael addition reactivity in the prediction of toxicity to Tetrahymena pyriformis. Chemosphere 2011, 85(6):1066-1074.

3. MOSES., is a C++ chemoinformatics software library that is developed and maintained by Molecular Networks GmbH, Erlangen, Germany (http://www. molecular-networks.com/software/moses/).

doi:10.1186/1758-2946-4-S1-O8

Cite this article as: Schwöbel et al.: Quantifying intrinsic chemical reactivity of molecular structural features for protein binding and reactive toxicity, using the MOSES chemoinformatics system. Journal of Cheminformatics 2012 4(Suppl 1):08. 